\title{
A Ten-year Experience of the Multidisciplinary Management of the Regional Failure of Nasopharyngeal Carcinoma in the Era of IMRT
}

\section{xiyin Guan}

shanghai proton and heavy ion center https://orcid.org/0000-0002-2150-8300

\section{Xing Xing}

fudan university shanghai cancer center

\section{Xiaoshen Wang}

Fudan University Shanghai Cancer Center

\section{Xiayun He}

Fudan University Shanghai Cancer Center

\section{Hongmei Ying}

Fudan University Shanghai Cancer Center

\section{Xueguan Lu}

Fudan University Shanghai Cancer Center

Chunying Shen

Fudan University Shanghai Cancer Center

Qunling Zhang

Fudan University Shanghai Cancer Center Jiaying Chen

Fudan University Shanghai Cancer Center Guohua Sun

Fudan University Shanghai Cancer Center

\section{Yu-Long Wang}

fudan university shanghai cancer center

\section{Yu Wang}

fudan university shanghai cancer center

\section{Tingting Xu}

Fudan University Shanghai Cancer Center

Chaosu Hu ( $\nabla$ hucsu62@126.com )

Fudan University Shanghai Cancer Center 
Keywords: nasopharyngeal carcinoma (NPC), intensity-modulated radiotherapy (IMRT), progression-free survival (PFS)

Posted Date: December 23rd, 2020

DOI: https://doi.org/10.21203/rs.3.rs-131844/v1

License: (c) (i) This work is licensed under a Creative Commons Attribution 4.0 International License. Read Full License 


\section{Abstract}

Objective: To evaluate clinical outcomes of cervical node recurrence/residual tumor of nasopharyngeal carcinoma (NPC) with treatment decisions made by a multidisciplinary treatment (MDT) team.

Methods: Between March 2010 and July 2019, patients who had previously undergone definitive intensity-modulated radiotherapy (IMRT) for nasopharyngeal carcinoma and presented with residual or recurrent cervical lymph nodes were enrolled in our study. Clinical features and treatment modalities were retrospectively reviewed, and survival outcomes were analyzed.

Results: A total of 183 patients were enrolled in the study, among whom 139 underwent neck dissection, and the remaining 44 were either considered not eligible for surgery or refused surgery. For the two groups of patients, the 3-year overall survival (OS) was $73.4 \%$ vs $36.8 \%(p=0.000)$, and the progression-free survival (PFS) was $57.1 \%$ vs $32.3 \%(p=0.004)$, respectively. Patients who underwent neck dissection had a significantly better clinical outcome and shorter latency between the prior treatment and detection of neck recurrence/residual tumor than those who did not.

Conclusion: For regional failure cases that have no contradictions for surgery and are predicted to benefit from surgery by the MDT team, neck dissection could be the best choice. It is important for an experienced MDT team to choose the optimum treatment modalities for patients for the cervical failure of NPC. Regular follow-up after prior treatment contributes to the early detection of regional failure and provides a chance for surgery.

\section{Introduction}

Nasopharyngeal carcinoma (NPC) is a comparatively rare malignancy and is more endemic in South China and Southeast Asia, with distinct pathological features and clinical characteristics from other head and neck cancers. Multidisciplinary treatment based on radiotherapy has been the mainstay of treatment for NPC. Over the past several decades, with the widely applied technology of IMRT, the clinical outcome of NPC has improved. The 5-year regional control of nasopharyngeal carcinoma can reach up to $90-95 \%$ 1-3. Thus, regional failure has received limited attention. The main strategy for regional failure is surgical dissection. However, several other treatments are also used by different cancer centers. A more standardized multidisciplinary treatment approach will not only help effectively control local recurrence but also reduce the risk of further progression. Patients with recurrence in a previously treated neck should still undergo surgery. For some specific cases, nonsurgical therapy may also be utilized when clinically appropriate. As one of the largest centers for head and neck cancer in China, our center has formed an experienced multidisciplinary treatment team for nasopharyngeal carcinoma and has accumulated sufficient experience in the treatment of the regional recurrence of NPC. In the current study, we reviewed treatment outcomes of patients who received definitive IMRT for nasopharyngeal carcinoma and were diagnosed with regional recurrence. Multidisciplinary treatment for the regional failure of 
nasopharyngeal carcinoma was evaluated, and a comprehensive analysis of the prognostic significance of patient clinical manifestations was performed.

\section{Materials And Methods}

\section{Patient characteristics}

From March 2010 to July 2019, patients who had previously undergone definitive IMRT with or without chemotherapy for nasopharyngeal carcinoma and presented with residual or recurrent cervical lymph nodes with no evidence of distant metastasis were included in our study. The patients' recurrence or residual tumors were diagnosed at Fudan University Shanghai Cancer Center, and patients received treatment at our center. We retrieved patient medical records and collected all detailed clinical characteristics, imaging, pathological reports, and treatment records of both prior treatment and recurrence/residual tumors. Exclusion criteria included the following: (1) the presence of a second primary malignancy, or NPC was a secondary malignancy; (2) postoperative pathology of the cervical node specimen showed no evidence of malignancy; and (3) insufficient clinical documentation. Tumors were staged according to the 7th edition of the American Joint Committee on Cancer/Union for International Cancer Control staging system.

\section{Treatment}

When pathologically or clinically diagnosed as cervical LN residual tumor or recurrence, patients were assessed by the multidisciplinary treatment team for head and neck cancer. Residual disease was defined as lymph nodes without complete regression by 3 or more months after primary therapy, and recurrent disease was defined as the reappearance of cervical lymph nodes after initial complete regression. Magnetic resonance imaging (MRI) and/or computerized tomography (CT) with contrast scans of the head and neck were performed to evaluate the extent of local regional disease and evaluated for surgical feasibility. For suspicious nodes that could not be diagnosed by an imaging study, fine needle aspiration (FNA) was performed. Patients who were eligible for neck dissection underwent either radical neck dissection (RND) or selective neck dissection (SND) based on the surgeon's decision and the patient's wishes. If neck dissection was not possible because of the node location, size, or invasion of adjacent tissues or if patients refused surgery, chemotherapy, radiotherapy or immunotherapy was administered. Patients who were eligible for reirradiation for cervical recurrence received IMRT, and the gross tumor volume (GTV) referred to the recurrence gross tumor or suspected residual tumor after resection. The total dose for recurrence was 60-66 grays (Gy). The choice of systemic therapy as individualized based on the patient's clinical condition. According to the NCCN guidelines, the preferred first-line regimen was cisplatin/gemcitabine. Other first-line recommendations included cisplatin/5-FU, cisplatin or carboplatin/docetaxel or paclitaxel, carboplatin/cetuximab, and gemcitabine/carboplatin. Single-agent therapies from the abovementioned regimens were also used for some patients.

\section{Statistical analysis}


All analyses were performed with SPSS software, version 26.0. Overall survival (OS), progression-free survival (PFS), regional recurrence-free survival (RRFS), local recurrence-free survival (LRFS), and distant metastasis-free survival (DMFS) were analyzed. Time-to-event statistics were calculated from the date of the diagnosis of recurrence to the event of interest. The Kaplan-Meier method was used to estimate survival rates, and the log-rank test was used to compare the differences. The hazard ratio (HR) and associated $95 \%$ confidence interval $(\mathrm{Cl})$ were calculated using the Cox proportional hazard model. Potential variables, including age, sex, the latency of nodal failure from prior treatment, staging classification at the initial presentation of NPC, whether disease was accompanied by synchronous local recurrence, residual or recurrent condition, $\mathrm{N}$ classification of nodal failure, extracapsular spread, cystic lymph nodes, levels and sides involved, and treatment modalities, were tested for their association with survival outcomes. Significant predictors identified in univariate analysis and factors that may have influenced clinical outcomes were included in multivariate analysis. The $\chi 2$ test was used to compare categorical variables. Independent t-tests were used to compare continuous variables. In all cases, a 2sided $p$ value $<0.05$ was considered significant.

\section{Results}

\section{Patient characteristics and the management of regional failure}

A total of 183 patients were enrolled in this study, among which 139 underwent resection, and the remaining 44 were either considered ineligible for surgery or refused surgery. Before treatment, all cases were discussed by our multidisciplinary treatment team, and for patients that were considered to have no contradictions for surgery and predicted to benefit from surgery by our MDT team, neck dissection was strongly recommended to the patients. Twenty-eight (15.3\%) patients had FNA pathology, and others were clinically diagnosed by the MDT team.

Clinical profiles of primary disease and prior treatment are listed in Table 1. All patients received a standard course of IMRT during prior treatment. The dose to the primary tumor and metastatic cervical nodes ranged from 66 to $70.4 \mathrm{~Gy}$ in $30-35$ fractions. A total dose of $60 \mathrm{~Gy}$ was delivered to the high-risk CTV and 50.4-54 Gy for low-risk CTV, respectively. A total of 179 (97.8\%) of the patients received platinum-based induction, concurrent, or adjuvant chemotherapy. Fourth-one (22.4\%) patients received a 2.0- to 6.0-Gy boost for lymph node residual disease in 1-3 fractions after the standard IMRT plan finished. Thirty-eight (20.8\%) patients had residual cervical lymph nodes 3 months after prior radiotherapy, and 27 (14.8\%) never achieved a complete response before resection.

All patients who underwent neck dissection were confirmed to have recurrence by postoperative pathology. The time from prior radiation therapy to the diagnosis of regional failure ranged from 2.4 to 105.6 months (median, 19.6 months) for the whole group. Forty-two (23.0\%) patients had synchronous primary site recurrence. Regarding the level of involved lymph node level involvement, 151 (82.5\%) patients had recurrence in the unilateral neck, and the other $32(17.5 \%)$ patients suffered from bilateral 
recurrence. According to our analysis of the lymph node level distribution, level llb (62.8\%) was the most common site of recurrence, followed by level Ila (35.5\%), level III (27.9\%), level V (16.4\%), level IV (8.7\%), and level I ( $8.2 \%)$. The number of patients with recurrence involving only one neck level, two levels, and more than two levels were $111(60.7 \%), 37(20.2 \%)$, and 35 (19.1\%), respectively. A total of $133(72.7 \%)$ patients were staged as rN1, $25(13.7 \%)$ as rN2, and the remaining $25(13.7 \%)$ as rN3. Additionally, based on the detailed review of imaging upon the diagnosis of recurrence, $104(56.8 \%)$ patients had extracapsular spread (ECS), and 104 (56.8\%) patients had cystic lymph nodes with internal necrosis. A total of $139(76.0 \%)$ patients underwent neck dissection, 56 (30.6\%) received chemotherapy, and 19 $(10.4 \%)$ patients received reirradiation for cervical recurrence. Details of the clinical features of recurrence are listed in Table 2.

\section{Treatment outcomes and prognostic analysis}

The median follow-up time after diagnosis of recurrence was 37.2 months (range, 6.1-118.2 months). During follow-up, 84 (45.9\%) patients died. Twenty-three (12.3\%) developed local recurrence, 56 (30.6\%) had regional relapse, and 24 (13.1\%) developed distant metastasis. The 3-year PFS and OS were 39.1\% and $64.1 \%$, respectively, and the 3-year RRFS and DMFS were $87.7 \%$ and $86.1 \%$, respectively.

For the 2 subgroups of patients who did or did not undergo neck dissection, the 3-year OS rates were $73.4 \%$ vs $36.8 \%$ ( $p=0.000$, Fig. $1 A)$, and the PFS rates were $57.1 \%$ vs $32.3 \%$ ( $p=0.004$, Fig. $1 B)$, respectively. Comparisons of clinical characteristics between patients in the neck dissection group and the non-neck dissection group are listed in Table 3. Patients who underwent neck dissection had a shorter interval between prior treatment and the diagnosis of neck recurrence than those who did not undergo neck dissection. The median interval of the non-neck dissection group was 30.8 (range: 6.0-96.0) months, compared to 15.9 (range: $2.4-105.6)$ months in the neck dissection group $(p<0.001)$. In the neck dissection group, only $16.5 \%$ of patients had synchronous local recurrence, which differed significantly from $43.2 \%$ of patients in the non-neck dissection group. Only $8.7 \%$ of patients in the neck dissection group had lymph nodes larger than $3 \mathrm{~cm}$, while in the non-neck dissection group, the proportion was $29.5 \%$. A total of $84.1 \%$ of patients who did not receive surgery had ECS, while in the surgery group, the proportion was only $48.2 \%$. Cystic lymph nodes did not differ between the two groups. For those who did not receive neck dissection, chemotherapy $(63.6 \%)$ and reirradiation $(20.5 \%)$ were administered. In the neck dissection group, $20.1 \%$ of patients received chemotherapy, and $7.2 \%$ received reirradiation before or after surgery.

Multiple clinical characteristics that might influence patient clinical outcomes were included in univariate analysis (Table 4), showing that age older than 50, not undergoing neck dissection, synchronous local recurrence, nodes with ECS, and receiving chemotherapy were associated with significantly worse PFS and OS. Multivariate analysis confirmed the improvement in OS conferred by surgery $(p=0.012)$. Age older than 50 years remained an independent prognostic factor for both PFS and OS. The details of multivariate analysis are shown in Table 5. 


\section{Discussion}

Multidisciplinary treatment for cervical lymph nodes or the recurrence of NPC after IMRT has seldom been reported in previous studies. Neck dissection is the preferred choice for the neck failure of NPC, with better treatment outcomes and a lower rate of treatment complications than reirradiation and/or chemotherapy ${ }^{4}$. In our study, it was remarkable that the treatment outcome was significantly better in those who received surgery, and the outcome was much worse for those who did not undergo surgical dissection. Therefore, evaluating patient eligibility for surgery is the primary task when dealing with the regional failure of NPC. The decision regarding treatment modality should be made by an experienced multidisciplinary team, which should be made up of not only radiation oncologists and radiologists but also, most importantly, surgeons specializing in head and neck surgery. In the MDT team in our center, when a patient had neck recurrence, we first evaluated the patient's general condition and excluded contradictions for surgery and then went through all the images before and after prior treatment and the images indicating node failure. After a detailed review of the patient's medical history, we evaluated whether the patient was eligible for surgery. Surgeons' experience is important in the decision regarding surgery. For more complicated cases, an experienced surgeon who has performed a considerable number of surgeries may a better chance of performing a total dissection and achieving better long-term survival. The selection of radical neck dissection or selective neck dissection was also discussed by the team based on the imaging studies of the patient. According to a former study by our center, selective neck dissection was demonstrated to be safe and effective for the treatment of neck residual disease of NPC for selected patients ${ }^{5}$. Considerations made by the MDT team are illustrated in Fig. 2. As long as the team considered the patient eligible for resection with no contradictions for surgery, we strongly suggested that the patient accept surgical interventions. Whether systemic treatment or radiotherapy might bring additional survival benefits before or after surgery was also determined by the MDT team.

Under certain circumstances (e.g., for patients who presented with synchronous local recurrence, patients who were predicted to hardly benefit from surgery, or patients with contraindications to surgery), a more conservative approach may be adopted. Over the past several decades, many potentially efficacious treatment options have emerged for the therapeutic management of regional recurrence in NPC patients who are not eligible for surgery ${ }^{6}$. Only a very limited proportion of our patients received reirradiation for regional recurrence and subsequently presented with severe complications secondary to cumulative irradiation injury, such as skin necrosis, soft tissue fibrosis, or brachial plexus injury. Chemotherapy was a comparatively more palliative strategy, as resistance to chemotherapy was common in cervical recurrence cases and the response rate was not satisfactory. Toxicity is often intolerable after several cycles and multiple lines of chemotherapy. Therefore, for patients with regional recurrence, indications for surgery must be carefully considered, and proper and personalized comprehensive treatment modalities should be identified. Our 10-year data indicated that having MDT discussions regarding each case and not neglecting a single case eligible for surgery, the best treatment choice would be selected for patients.

It is notable that according to our data and consistent with other studies ${ }^{7}$, the distribution of recurrent or residual nodes was not as extensive as in primary NPC cases; $60.7 \%$ of patients presented with a 
comparatively limited range of recurrence, involving only one level on one side, and $86.3 \%$ presented with lymph nodes with diameters less than $3 \mathrm{~cm}$. Level II (84.2\%) was the most common site of nodal recurrence. Prior radical radiotherapy may have impaired the cervical lymphatic drainage function, thereby reducing the secondary cervical metastasis of the tumor. The interval between the end of prior treatment and the diagnosis of neck recurrence was much shorter in the neck dissection group than in the non-neck dissection group, suggesting that patients with indications for neck dissection are more likely to be diagnosed at an early stage of disease. A relatively large proportion of patients went on for routine follow-up after prior treatment, which occurred every 3 months within the first 2 years and every 6 months in the 3rd to 5 th years after treatment. The peak of recurrence diagnosis occurred at approximately 20 months, ranging from 2.4 months to 105 months, so intensive follow-up should always be recommended for NPC patients who finish definitive prior treatment. It was also proposed by our previous study that close follow-up is still important for the early detection of recurrence in years $3-4$ after treatment ${ }^{8}$. Those who were considered to benefit less from surgery or lost the chance for surgery may have not had regular follow-up until the clinical presentation was too severe, for example, when the recurrent lymph nodes might have been too large, or might have too extensive involvement of the adjacent structures, making a total dissection difficult to perform. Therefore, for nasopharyngeal carcinoma patients, regular follow-up after treatment completion is crucial, and the early detection of regional recurrence may provide a better chance of complete resection.

Univariate analysis showed that age older than 50, synchronous local recurrence, extracapsular spread, not receiving surgery and receiving chemotherapy were related to a significantly worse prognosis. Previous studies ${ }^{9}$ reported similar results, suggesting that younger patients (age $<40-50$ years) had a better prognosis for cervical recurrence. Older patients tend to have a lower KPS and worse heart or lung function, making surgery or even other comprehensive treatment modalities intolerable. Therefore, treatment selection for older patients should be performed more cautiously. Cervical recurrence with concurrent primary site recurrence in the nasopharynx is not rare and might originate from specific malignant cells with resistance to prior chemoradiotherapy. The treatment decision for this group of patients varies and is still based on each patient's specific condition. For those with a limited range of local recurrence that could be managed by endoscopic surgery or reirradiation and cervical recurrence that could benefit from surgery, the separate management of recurrence in both sites might be effective. It was suggested in a study from Hong Kong that neck dissection is efficacious in patients with nasopharyngeal carcinoma with nodal failure with or without synchronous local failure ${ }^{10}$. Systemic treatment should also be considered for some patients. However, the control rate of local recurrence is not always satisfactory, with considerable rates of nasopharyngeal necrosis and hemorrhage shortly after treatment, especially in patients receiving reirradiation. In this case, each case should be discussed in detail by an MDT team to select the best treatment. Extracapsular spread is usually considered related to worse outcomes ${ }^{11,12}$, regardless of primary or recurrent nodes. Extensive soft tissue and muscle invasion are barriers to complete resection, and adjuvant local reirradiation or chemotherapy might be indispensable for effective regional control. However, a recent study proposed that within a group of 46 patients who received neck dissection after the diagnosis of nodal recurrence, ECS was not correlated 
with a worse survival. They proposed that an absolute number of positive lymph nodes greater than five and a lymph node density greater than $20 \%$ were potentially useful prognostic factors ${ }^{13}$. Patients who received chemotherapy were usually not eligible for surgery, so it could be speculated that chemotherapy is related to a worse prognosis, as shown in the univariate analysis. Multivariate analysis revealed that surgery and age older than 50 were the only 2 independent prognostic factors for OS, suggesting the dominant role of surgery in the management of cervical recurrence.

Several other studies have demonstrated that patients with cervical node residual disease after prior radical irradiation may have a better prognosis than patients who present with node recurrence. This was also observed in our colleague Zhang's former study based on conventional radiotherapy ${ }^{14}$. In the current study, however, patients with cervical node residual disease did not have better survival than those with cervical node recurrence, even though the range of the cervical residual disease was usually limited, and there was a greater chance of neck total resection. A review of the data showed that there was a larger proportion of N3 patients in the residual disease group than in the recurrence group ( $51.8 \%$ vs. $24.3 \%)$, so the possible survival advantages of patients with residual disease might be offset by the larger proportion of N3 patients, who obviously had worse clinical outcomes. In addition, in our study, all patients received IMRT as prior treatment for primary NPC. Compared with conventional radiotherapy, IMRT achieves better survival, with benefits of a better dose distribution, i.e., better dose heterogeneity within the target volume and a lower dose to organs at risk (OAR). Thus, residual lymph nodes after IMRT might usually be comparatively more radiation resistant. In our study, $22.4 \%$ of patients received a boost for obvious lymph node residual disease when the IMRT course finished, and the rate was much higher than the reported rate of $8.3 \%$ in general in a previous study from our center ${ }^{1}$.

IMRT technology has been used at our center for NPC patients since 2005. Most patients who received prior treatment returned to our center for follow-up, and subsequent treatment for treatment failure was also received here. A total of 355 patients with neck residual disease or the recurrence of NPC after 2D-RT who underwent radical neck dissection over a 10-year period from 1998 to 2007 were reported before. The 3- and 5-year overall survival rates were $54.1 \%$ and $26.0 \%$, respectively. In this study, within a decade, the survival of nasopharyngeal carcinoma improved, and cases of failure decreased significantly. Only 139 patients underwent neck dissection at our center. This group of patients achieved much higher 3-and 5 -year overall survival rates of $73.4 \%$ and $52.8 \%$, respectively. In addition to improvements in radiation techniques, advances in systemic treatment and more accurate imaging techniques, such as MRI and PET/CT ${ }^{15,16}$, also contributed to better clinical outcomes. Moreover, new advances in systemic therapy, especially immunotherapy, have provided promising preliminary results for recurrent and metastatic nasopharyngeal carcinoma ${ }^{17-19}$. Including immunotherapy in multidisciplinary treatment is a new trend, and the best combination of surgery, radiotherapy and systemic therapy should be further examined and discussed.

The retrospective nature was a major limitation of our study. Patients' conditions varied, and strategies for each case were determined by the MDT team based on their clinical features. Based on the current 
findings and experiences, we will perform further prospective studies focusing on developing a clearer standard that could allow patients to benefit from surgery and identify the best comprehensive treatment strategies for patients.

In conclusion, for recurrent or residual nodes of NPC after prior chemoradiotherapy, radical neck dissection could be the best choice after a comprehensive multidisciplinary evaluation of the patients' condition. Regular, close follow-up after treatment and access to an experienced MDT team to make optimal treatment decisions for patients are key components to a better clinical outcome for the regional failure of NPC.

\section{Abbreviations}

NPC, Nasopharyngeal carcinoma; MDT, multidisciplinary treatment; PFS, progression free survival; FNA, fine needle aspiration; IMRT, intensity-modulated radiotherapy; MRI, magnetic resonance imaging; PET-CT, positron emission tomography; $C T$, computerized tomography; FNA, fine needle aspiration; RND, radical neck dissection; SND, selective neck dissection; GTV, gross tumor volume; Gy, grays; OS, overall survival; RRFS, regional recurrence-free survival; LRFS, local recurrence-free survival; DMFS, distant metastasisfree survival; $\mathrm{HR}$, hazard ratio; $\mathrm{Cl}$, confidence interval; $\mathrm{CTV}$, clinical target volume; $\mathrm{ECS}$, extra-capsular spread.

\section{Declarations}

\section{- Ethical Approval and Consent to participate}

The retrospective study was approved by the Ethical Review Board of Fudan University Shanghai Cancer Center.

\section{- Consent for publication}

All authors have read and approved the final manuscript for publication.

\section{- Availability of supporting data}

Not applicable

\section{- Competing interests}

The authors declare that they have no competing interests.

\section{- Funding}


The study was funded by the National Key Technologies Research and Development Program on Prevention and Control of Chronic Non-communicable Diseases (Grant No.2018YFC1313204) and Shanghai Municipal Health Commission (Grant No.20204Y0283). All the grant sponsors had no involvement in the study design, data analysis, preparation, or writing of this manuscript.

\section{- Authors' contributions}

Tingting Xu, Chaosu Hu developed the study design and coordination, and helped to review the manuscript, Xiyin Guan and Xing Xing carried out the data collection and the statistical analysis, and drafted the manuscript, Xiaoshen Wang , Xiayun He, Hongmei Ying , Xueguan Lu , Chunying Shen, Qunling Zhang, Jiaying Chen, Guohua Sun, Yu-Long Wang , Yu Wang ,recruit patients and provide patients care. All authors read and approved the final manuscript.

\section{- Acknowledgements}

We acknowledge the work of colleagues in the department of Radiation Oncology, Head and Neck surgery, and Medical oncology, , Fudan University Shanghai Cancer Center related to this article.

\section{References}

1. Ou X, Zhou X, Shi Q, et al. Treatment outcomes and late toxicities of 869 patients with nasopharyngeal carcinoma treated with definitive intensity modulated radiation therapy: New insight into the value of total dose of cisplatin and radiation boost. Oncotarget. 2015;6(35):38381-38397. doi:10.18632/oncotarget.5420

2. Setton J, Han J, Kannarunimit D, et al. Long-term patterns of relapse and survival following definitive intensity-modulated radiotherapy for non-endemic nasopharyngeal carcinoma. Oral Oncol. 2016;53(5):67-73. doi:10.1016/j.oraloncology.2015.11.015

3. Tian YM, Liu MZ, Zeng L, et al. Long-term outcome and pattern of failure for patients with nasopharyngeal carcinoma treated with intensity-modulated radiotherapy. Head Neck. 2019;41(5):1246-1252. doi:10.1002/hed.25545

4. Chua DTT, Wei WI, Sham JST, Cheng ACK, Au G. Treatment outcome for synchronous locoregional failures of nasopharyngeal carcinoma. Head Neck. 2003;25(7):585-594. doi:10.1002/hed.10242

5. Chen JY, Zhang L, Ji QH, et al. Selective neck dissection for neck residue of nasopharyngeal carcinoma: A prospective study. J Cranio-Maxillofacial Surg. 2015;43(8):1571-1576. doi:10.1016/j.jcms.2015.06.037

6. Lewis CM, Nurgalieva Z, Sturgis EM, Lai SY, Weber RS. Improving patient outcomes through multidisciplinary treatment planning conference. Head Neck. 2016;38 Suppl 1:E1820-5. doi:10.1002/hed.24325

7. Liu YP, Li H, You R, et al. Surgery for isolated regional failure in nasopharyngeal carcinoma after radiation: Selective or comprehensive neck dissection. Laryngoscope. 2019;129(2):387-395. 
doi:10.1002/lary.27317

8. Xu T, Zhou X, Shen C, Hu C. Suggestions for surveillance and radiation strategy in nasopharyngeal carcinoma treated with IMRT: Based on hazard-rate and patterns of recurrence. Oral Oncol. 2018;76(December 2017):61-67. doi:10.1016/j.oraloncology.2017.11.022

9. Wang S, Lou J, Chen J, Zhang S, Guo L. Salvage surgery for neck residue or recurrence of nasopharyngeal carcinoma after primary radiotherapy: options of surgical methods and regions. World J Surg Oncol. 2016:1-6. doi:10.1186/s12957-016-0822-8

10. Tsang RKY, Chung JCK, Ng YW, et al. Efficacy of neck dissection for locoregional failures versus isolated nodal failures in nasopharyngeal carcinoma. Head Neck. 2012;34(5):638-642. doi:10.1002/hed.21781

11. Chan YW, Fun Lee VH, Yu Chow VL, Howe To VS, Wei WI. Extracapsular lymph node spread in recurrent nasopharyngeal carcinoma. Laryngoscope. 2011;121(12):2576-2580. doi:10.1002/lary.22380

12. Lin CY, Tsai ST, Jin YT, Yang MW, Yeh IC, Hsiao JR. Outcome of surgical management of persistent or recurrent neck mass in patients with nasopharyngeal carcinoma after radiotherapy. Eur Arch OtoRhino-Laryngology. 2008;265(SUPPL. 1):69-74. doi:10.1007/s00405-007-0552-1

13. Yeung DCM, Yeung Z, Wong EWY, Vlantis AC, Chan JYK. Neck lymph node status on survival of regionally recurrent or persistent nasopharyngeal carcinoma. Sci Rep. 2020;10(1):1-8. doi:10.1038/s41598-020-62625-4

14. Zhang L, Zhu YX, Wang Y, Huang CP, Wu Y, Ji QH. Salvage surgery for neck residue or recurrence of nasopharyngeal carcinoma: A 10-year experience. Ann Surg Oncol. 2011;18(1):233-238. doi:10.1245/s10434-010-1292-9

15. Yao M, Smith RB, Graham MM, et al. The role of FDG PET in management of neck metastasis from head-and-neck cancer after definitive radiation treatment. Int J Radiat Oncol Biol Phys. 2005;63(4):991-999. doi:10.1016/j.jijrobp.2005.03.066

16. Toh ST, Yuen HW, Goh YH, Goh CHK. Evaluation of recurrent nodal disease after definitive radiation therapy for nasopharyngeal carcinoma: diagnostic value of fine-needle aspiration cytology and CT scan. Head Neck. 2007;29(4):370-377. doi:10.1002/hed.20526

17. Ma BBY, Lim W-T, Goh B-C, et al. Antitumor Activity of Nivolumab in Recurrent and Metastatic Nasopharyngeal Carcinoma: An International, Multicenter Study of the Mayo Clinic Phase 2 Consortium (NCl-9742). J Clin Oncol Off J Am Soc Clin Oncol. 2018;36(14):1412-1418. doi:10.1200/JCO.2017.77.0388

18. Hsu C, Lee S-H, Ejadi S, et al. Safety and Antitumor Activity of Pembrolizumab in Patients With Programmed Death-Ligand 1-Positive Nasopharyngeal Carcinoma: Results of the KEYNOTE-028 Study. J Clin Oncol Off J Am Soc Clin Oncol. 2017;35(36):4050-4056. doi:10.1200/JCO.2017.73.3675

19. Fang W, Yang Y, Ma Y, et al. Camrelizumab (SHR-1210) alone or in combination with gemcitabine plus cisplatin for nasopharyngeal carcinoma: results from two single-arm, phase 1 trials. Lancet 


\section{Tables}

Table 1

Clinical features of prior treatment

\begin{tabular}{|c|c|c|c|}
\hline Characteristics & & Number & Percentage (\%) \\
\hline \multirow[t]{2}{*}{ Gender } & Male & 150 & 82.0 \\
\hline & Female & 33 & 18.0 \\
\hline \multirow[t]{3}{*}{ Staging } & II & 31 & 16.9 \\
\hline & III & 77 & 42.1 \\
\hline & IV & 75 & 41.0 \\
\hline \multirow[t]{4}{*}{ N staging } & No & 5 & 2.7 \\
\hline & $\mathrm{N} 1$ & 54 & 29.5 \\
\hline & $\mathrm{N} 2$ & 72 & 39.3 \\
\hline & N3 & 52 & 28.4 \\
\hline \multirow[t]{3}{*}{ Lymph node size $(\mathrm{cm})$} & $<3$ & 74 & 40.4 \\
\hline & $3-6$ & 88 & 48.1 \\
\hline & $\geq 6$ & 21 & 11.5 \\
\hline \multirow[t]{2}{*}{ ECS } & Yes & 114 & 62.3 \\
\hline & No & 65 & 37.7 \\
\hline \multirow[t]{2}{*}{ Necrosis } & Yes & 59 & 32.2 \\
\hline & No & 118 & 67.8 \\
\hline \multirow[t]{2}{*}{ Boost to node residue } & Yes & 41 & 22.4 \\
\hline & No & 142 & 77.6 \\
\hline
\end{tabular}


Table 2

Clinical features of recurrence

\begin{tabular}{|c|c|c|c|}
\hline Characteristics & & Number & Percentage (\%) \\
\hline \multirow[t]{2}{*}{ Age } & Median & 52 & \\
\hline & Range & $29-75$ & \\
\hline \multirow[t]{2}{*}{ Interval between prior RT and recurrence } & Median & 19.6 & \\
\hline & Range & $2.4-105.6$ & \\
\hline \multirow[t]{3}{*}{ rN staging } & $\mathrm{rN} 1$ & 133 & 72.7 \\
\hline & rN2 & 25 & 13.7 \\
\hline & rN3 & 25 & 13.7 \\
\hline \multirow[t]{2}{*}{ Sides } & Unilateral & 151 & 82.5 \\
\hline & Bilateral & 32 & 17.5 \\
\hline \multirow[t]{2}{*}{ Synchronous local recurrence } & Yes & 42 & 23.0 \\
\hline & No & 141 & 77.0 \\
\hline \multirow[t]{2}{*}{ Lymph node size } & $<3$ & 157 & 86.3 \\
\hline & $\geq 3$ & 26 & 13.7 \\
\hline \multirow[t]{2}{*}{ Levels involved } & 1 & 111 & 60.7 \\
\hline & $>1$ & 72 & 39.3 \\
\hline \multirow[t]{2}{*}{ ECS } & Yes & 104 & 56.8 \\
\hline & No & 79 & 43.2 \\
\hline \multirow[t]{2}{*}{ Cystic lymph nodes } & Yes & 104 & 56.8 \\
\hline & No & 79 & 43.2 \\
\hline \multirow[t]{2}{*}{ Neck dissection } & Yes & 139 & 76.0 \\
\hline & No & 44 & 24.0 \\
\hline \multirow[t]{2}{*}{ chemotherapy } & Yes & 56 & 30.6 \\
\hline & No & 127 & 69.4 \\
\hline \multirow[t]{2}{*}{ Re-irradiation } & Yes & 19 & 10.4 \\
\hline & No & 164 & 89.6 \\
\hline
\end{tabular}


Table 3

Comparison between surgery group vs non-surgery group

\begin{tabular}{|c|c|c|c|c|}
\hline & & Non-surgery $(n=139)$ & Surgery $(n=44)$ & $P$ value \\
\hline Age & Median & $53(29-73)$ & $51(30-75)$ & 0.210 \\
\hline \multirow[t]{2}{*}{ Interval from prior treatment } & Median & 30.8 & 15.9 & 0.000 \\
\hline & Range & $6.0-96.0$ & $2.4-105.6$ & \\
\hline \multirow[t]{3}{*}{$\mathrm{N}$ stage at nodal failure } & rN1 & $28(63.6)$ & 105(75.5) & 0.304 \\
\hline & rN2 & $8(18.2)$ & $17(12.2)$ & \\
\hline & rN3 & $8(18.2)$ & $17(12.2)$ & \\
\hline \multirow[t]{2}{*}{ Sides } & Unilateral & $34(77.4)$ & $117(84.2)$ & 0.294 \\
\hline & Bilateral & $10(22.7)$ & $22(15.8)$ & \\
\hline \multirow[t]{2}{*}{ Synchronous local recurrence } & Yes & 19(43.2) & $23(16.5)$ & 0.000 \\
\hline & No & $25(56.8)$ & 116(83.5) & \\
\hline \multirow[t]{2}{*}{ Maximum lymph node size } & $<3$ & $31(70.5)$ & 126(91.3) & 0.000 \\
\hline & $\geq 3$ & 13(29.5) & $11(8.7)$ & \\
\hline \multirow[t]{2}{*}{ Lymph node levels involved } & 1 & 19(43.2) & $92(66.2)$ & 0.006 \\
\hline & $>1$ & $25(56.8)$ & $47(33.8)$ & \\
\hline \multirow[t]{2}{*}{ Extracapsular spread } & Yes & $37(84.1)$ & $67(48.2)$ & 0.000 \\
\hline & No & $7(15.9)$ & 72(51.8) & \\
\hline \multirow[t]{2}{*}{ Cystic lymph nodes } & Yes & $29(65.9)$ & $75(54.0)$ & 0.163 \\
\hline & No & 15(34.1) & $64(46.0)$ & \\
\hline \multirow[t]{2}{*}{ Chemotherapy } & Yes & $28(63.6)$ & $28(20.1)$ & 0.000 \\
\hline & No & $16(36.4)$ & 111(79.9) & \\
\hline \multirow[t]{2}{*}{ Re-irradiation } & Yes & $9(20.5)$ & $10(7.2)$ & 0.012 \\
\hline & No & $35(79.5)$ & $129(92.8)$ & \\
\hline
\end{tabular}


Table 4

Univariate analysis

\begin{tabular}{|c|c|c|c|c|c|}
\hline Variables & & 3-year PFS (\%) & $P$ value & 3-year OS (\%) & $P$ value \\
\hline \multirow[t]{2}{*}{ Age } & $<50$ & $45.0 \%$ & 0.039 & $69.8 \%$ & 0.009 \\
\hline & $>50$ & $33.1 \%$ & & $58.1 \%$ & \\
\hline \multirow[t]{2}{*}{ rN stage } & $\mathrm{rN} 1-2$ & $40.5 \%$ & 0.228 & $67.2 \%$ & 0.121 \\
\hline & rN3 & $29.9 \%$ & & $44.5 \%$ & \\
\hline \multirow[t]{2}{*}{ Synchronous local recurrence } & Yes & $42.7 \%$ & 0.036 & $41.4 \%$ & 0.000 \\
\hline & No & $55.0 \%$ & & $70.1 \%$ & \\
\hline \multirow[t]{2}{*}{ Lymph node size (cm) } & $<3$ & $42.6 \%$ & 0.093 & $67.2 \%$ & 0.086 \\
\hline & $\geq 3$ & $18.3 \%$ & & $45.2 \%$ & \\
\hline \multirow[t]{2}{*}{ ECS } & Yes & $29.4 \%$ & 0.007 & $55.4 \%$ & 0.005 \\
\hline & No & $52.3 \%$ & & $75.9 \%$ & \\
\hline \multirow[t]{2}{*}{ Residue } & Yes & $29.6 \%$ & 0.712 & $55.6 \%$ & 0.407 \\
\hline & No & $41.0 \%$ & & $66.0 \%$ & \\
\hline \multirow[t]{2}{*}{ Neck dissection } & Yes & $57.1 \%$ & 0.004 & $73.4 \%$ & 0.000 \\
\hline & No & $36.8 \%$ & & $32.3 \%$ & \\
\hline \multirow[t]{2}{*}{ chemotherapy } & Yes & $37.9 \%$ & 0.003 & $50.4 \%$ & 0.000 \\
\hline & No & $58.8 \%$ & & $71.4 \%$ & \\
\hline \multirow[t]{2}{*}{ Re-irradiation } & Yes & $35.1 \%$ & 0.641 & $73.0 \%$ & 0.880 \\
\hline & No & $39.6 \%$ & & $63.0 \%$ & \\
\hline \multirow[t]{2}{*}{ Interval less than 24 months } & Yes & $40.0 \%$ & 0.854 & $67.1 \%$ & 0.886 \\
\hline & No & $37.4 \%$ & & $60.4 \%$ & \\
\hline
\end{tabular}


Table 5

Multivariate analysis

\begin{tabular}{|llll|}
\hline \multicolumn{1}{|l}{ Variables } & P value & HR \\
\hline PFS & Age $(\geq$ 50y v.s. $<50 y)$ & 0.032 & 1.489 \\
\cline { 2 - 4 } & Synchronous local recurrence & 0.425 & 0.824 \\
\hline ECS & 0.147 & 0.733 \\
\hline Surgery & 0.338 & 1.278 \\
\hline Chemotherapy & 0.361 & 0.792 \\
\hline OS & 0.757 & 0.921 \\
\hline Age ( $\geq$ 50y v.s. <50y) & 0.007 & 1.851 \\
\hline Synchronous local recurrence & 0.097 & 0.631 \\
\hline ECS & 0.324 & 0.770 \\
\hline Surgery & 0.012 & 2.098 \\
\hline Chemotherapy & 0.339 & 0.747 \\
\hline Lymph node size & 0.725 & 0.897 \\
\hline
\end{tabular}

Figures 

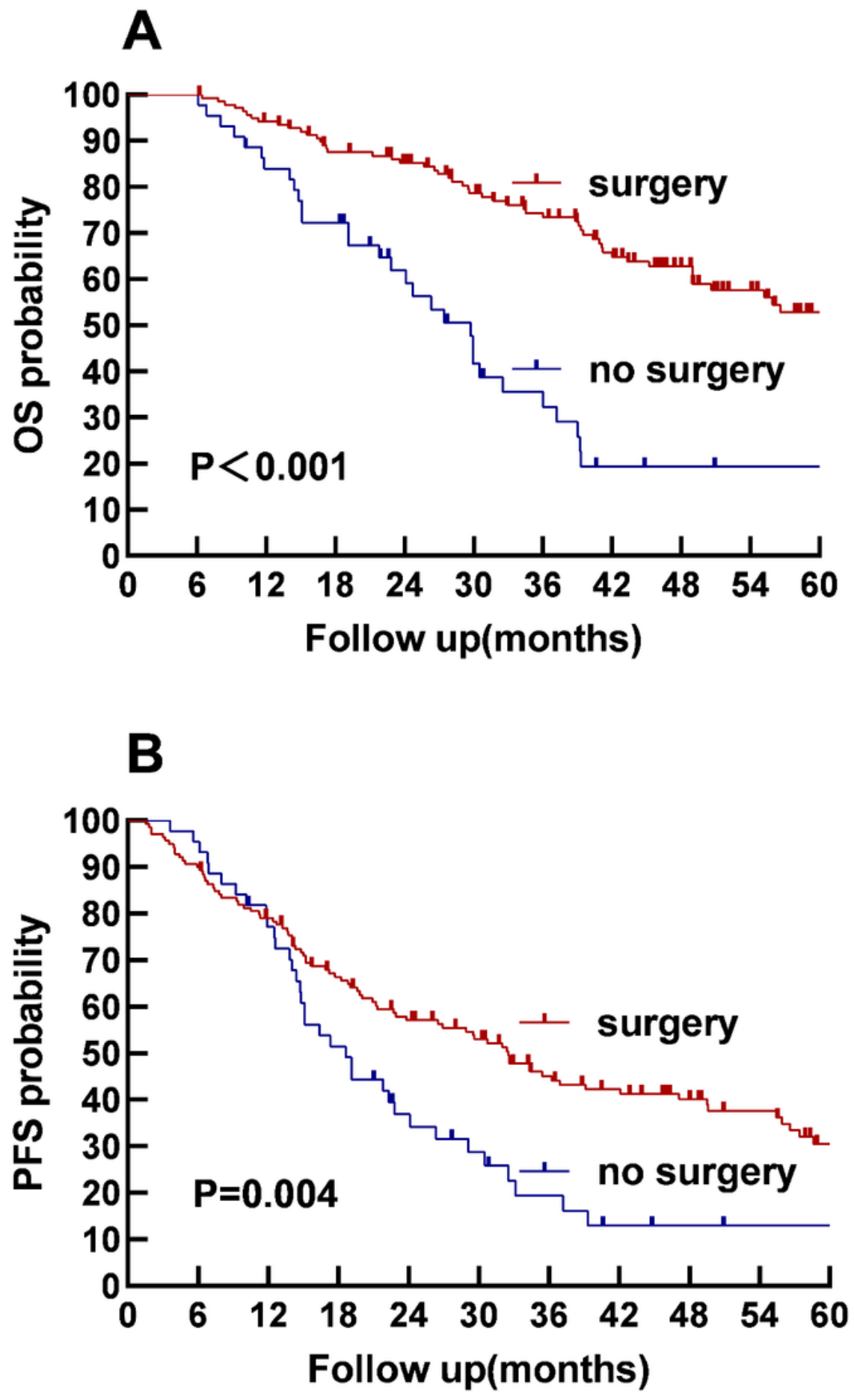

Figure 1

Survival of patients in surgery group and non-surgery group. (A) Overall-survival (B) Progression-free survival. 


\section{Multidisciplinary Treatment Considerations for Regional Failure}

Diagnosis of

recurrence/residue

- Physical examination

- Imaging: MRI, CT, PET/CT

- Biopsy/FNA

- EBV DNA

- Synchronous local recurrence

- Distant metastasis
Prior treatment

- Staging

- Radiation techniques

- Multidisciplinary treatment

- Dose to targets and OARs

- Late complications
Patient condition

- Age

- Performance score

- Comorbidities

- Patients' own wishes

\section{Resectability}

- Levels involved

- Sides

- Numbers of nodes

- ECS

- Range of invasion into muscles, soft tissue, skin and carotid vessel

\section{Multidisciplinary}

Treatment

- Neck dissection

- Chemotherapy

- Re-irradiation

- Immunotherapy

- Clinical trials

\section{Figure 2}

Multidisciplinary treatment considerations for cervical failure 